\section{A CROSS-RESISTANCE OF \\ PYRICULARIA ORYZAE CAVARA TO KASUGAMYCIN AND BLASTICIDIN S}

\author{
Hisashi Sakurai and Hisashi Naito \\ Agricultural Chemicals Inspection Station, \\ Ministry of Agriculture and Forestry, \\ Kodaira-shi, Tokyo, Japan
}

(Received for publication September 3, 1976)

A cross resistance of pathogenic organisms to various chemicals has been increasing over a long period of time causing serious problems in the field of antibacterial drugs and veterinary drugs. Recently, it was reported that some phytopathogenic bacteria and fungi resistant to antibiotics and synthetic fungicides were isolated from diseased plants in the field and that distribution of resistant strains shows a rapid annual increase. There were reports that the resistance to benomyl in some phytopathogenic fungi coincides with resistance to the other benzimidazole derivatives and also to thiophanate methyl. ${ }^{1,2,3,4)}$ We also reported that kasugamycin-resistant strains of rice blast fungus (Pyricularia oryzae CAVARA) had cross resistance to blasticidin $\mathrm{S}$ and polyoxin D. ${ }^{5)}$

Minimal inhibitory concentration (MIC) values for $P$. oryzae obtained from diseased rice plant from 1972 through 1975 were studied, and a method for establishing the appropriate MIC values for grouping the isolates into sensitive and resistant strains was described in the previous paper. ${ }^{6)}$

In the present study, MIC values for each kasugamycin and blasticidin $\mathrm{S}$ were determined by streak culture on agar using two-fold serial dilutions of antibiotics. For MIC of antibiotic assayed with $P$. oryzae, the rice plant juice medi$\mathrm{um}^{5)}$ was used. In order to determine MIC value against test isolates, mycelial fragments of each test isolates were placed on agar and kept for 48 hours at $28^{\circ} \mathrm{C}$.

The following method was adopted to determine the cross-resistance to kasugamycin and blasticidin S. To minimize the effects of various variable conditions, MIC values of isolates studied over 2 years were pooled and the correlogram of MIC values between kasugamycin and blasticidin $\mathrm{S}$ of $P$. oryzae were plotted in a
Fig. 1. Correlogram of MIC values between kasugamycin and blasticidin S of Pyricularia oryzae. (218 isolates)

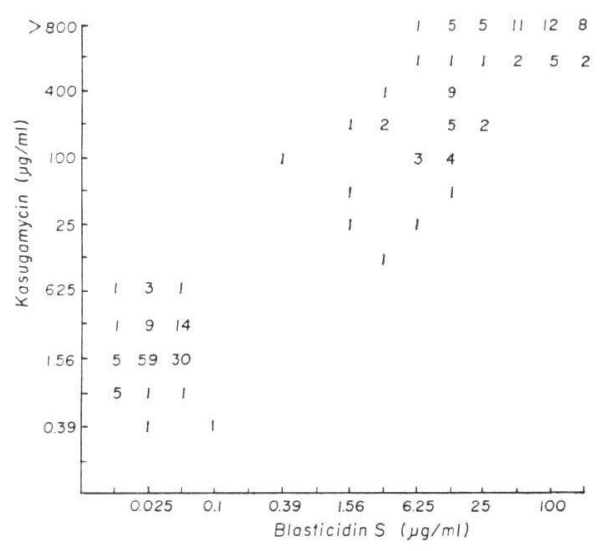

single figure (Fig. 1). The in vitro activity of kasugamycin and blasticidin $\mathrm{S}$ against $P$. oryzae clearly demonstrates that the resistance to kasugamycin in isolates of $P$. oryzae coincides with resistance to blasticidin $\mathrm{S}$.

The relation between MIC of antibiotics for isolates of P. oryzae and the therapeutic effect on rice blast of the application of kasugamycin and blasticidin $\mathrm{S}$ was investigated. A method of determinating the therapeutic effect on rice blast of the antibiotic was described in the previous paper. $^{5)}$ As shown in Table 1, the results indicated that the relation between MIC values of antibiotics in isolates of $P$. oryzae and therapeutic effect of rice blast correlated with each other, although there is no relation in chemical structure between kasugamycin and blasticidin $\mathrm{S}$. The in vitro and in vivo activity of kasugamycin and blasticidin $\mathrm{S}$ against $P$. oryzae are of very interest, showing that kasugamycin-resistant strains of $P$. oryzae also were cross-resistance to blasticidin $\mathbf{S}$.

A detailed comparison of the sensitivity and accuracy of both the MIC test and the therapeutic effect of the antibiotics on rice blast produced with many isolates of $P$. oryzae has not been made. Furthermore the correlogram of MIC values shown in this study (Fig. 1) might not be applicable at the concentrations of antibiotics used in the fields. However, when used together with the knowledge of a method of application and of the pharmacokinetics of antibiotics, the correlogram of MIC values between kasugamycin and blasticidin $\mathrm{S}$ might contribute information that would 
Table 1. The relation between MIC of antibiotics in isolates of Pyricularia oryzae and therapeutic effect on rice blast of kasugamycin and blasticidin $\mathrm{S}$ in the rice seedlings.

\begin{tabular}{c|c|c|c|c}
\hline \multirow{2}{*}{$\begin{array}{c}\text { Designation of } \\
\text { isolates }\end{array}$} & \multicolumn{2}{|c|}{ MIC $(\mu \mathrm{g} / \mathrm{ml})$} & \multicolumn{2}{c}{ Therapeutic effect of rice blast* } \\
\cline { 2 - 3 } $71-3$ & KsM & BcS & KsM $(20 \mu \mathrm{g} / \mathrm{ml})$ & $\mathrm{BcS}(10 \mu \mathrm{g} / \mathrm{ml})$ \\
\hline $47-\mathrm{Y}-3$ & 200 & 25 & 13.4 & 0.2 \\
$47-\mathrm{Y}-15$ & 200 & 100 & 8.5 & 27.6 \\
$47-Y-35$ & 100 & 12.5 & 47.8 & 0 \\
P- 2 & 0.78 & $<0.19$ & 99.0 & 91.0 \\
Hoku 373 & 0.78 & $<0.19$ & 98.6 & 99.0 \\
\hline
\end{tabular}

* Percent decrease in number of lesions per leaf by application of the antibiotic.

KsM; Kasugamycin. BcS; Blasticidin S.

be useful for application of antibiotics in the field.

\section{References}

1) Bollen, G. J. \& G. Scholten: Acquired resistance to benomyl and some other systemic fungicides in a strain of Botrytis cinerea in cyclamen. Neth. J. Path. 77: 83 90, 1971

2) Bollen, G. J.: Resistance to benomyl and some chemically related compounds in strains of Penicillium species. Neth. J. Path. 77: 187 193, 1971

3) Schooley, J. B. \& B. H. MacNeil: A compari- son of the modes of action of three benzimidazoles. Phytopathol. 61: 816 819, 1971

4) VARGas, J. M.: A benzimidazole resistant strain of Erysiphe graminis. Phytopathol. 63: 1366 1368, 1973

5) Sakurai, H.; H. Naito \& K. Yoshida: Studies on cross resistance to antifungal antibiotics in kasugamycin-resistant strains of Pyricularia oryzae Cavara. Bull. Agr. Chem. Inspect. Stn. No. 15: 82 91, 1975

6) Sakurai, H.; H. Naito \& S. Fujita: Sensitivity distribution of phytopathogenic bacteria and fungi to antibiotics. J. Antibiotics 29: 1230 1236, 1976 